J Res Pers. 2014 December 1; 53: 22-30. doi:10.1016/j.jrp.2014.08.002.

\title{
Does mindfulness attenuate thoughts emphasizing negativity, but not positivity?
}

\author{
Laura G. Kiken and \\ University of North Carolina, Chapel Hill, NC, USA \\ Natalie J. Shook \\ West Virginia University, Morgantown, WV, USA
}

\begin{abstract}
The current research investigated whether mindfulness is differentially associated with thoughts that emphasize positive or negative valence. In Study 1, trait mindfulness was inversely associated with negative rumination but unassociated with positive rumination, controlling for state affect. In Study 2, participants completed either a mindful breathing meditation or a comparable control exercise, followed by a thought listing while viewing affective images. Compared to the control condition, the mindfulness condition listed proportionately fewer negative thoughts, particularly in response to negative images, and more non-valenced thoughts. The conditions did not differ in their proportions of positive thoughts. These results suggest that mindfulness may attenuate thoughts that emphasize negativity but not those that emphasize positivity.
\end{abstract}

\section{Keywords}

mindfulness; cognition; thought; bias; rumination; valence; negative; positive

\subsection{Introduction}

Mindfulness involves using awareness to direct attention to current experiences as they unfold from moment to moment in a receptive or nonjudgmental way (cf. Kabat-Zinn, 1990). Mindfulness has been studied as a trait, in terms of individual differences in the tendency to be mindful in daily life (e.g., Baer, Smith, Hopkins, Krietemeyer, \& Tooney, 2006; Brown \& Ryan, 2003) and as a practicable state in mindfulness meditation and meditation-based interventions (cf. Brown, Ryan, \& Creswell, 2007). Much of this evidence base concerns the benefits of mindfulness for preventing and reducing psychological distress and disorder, both in clinical and nonclinical populations (Keng et al., 2011; Khoury et al., 2013). Recent investigations have started to investigate potential mechanisms for such

\footnotetext{
(c) 2014 Elsevier Inc. All rights reserved.

Address correspondence to: Laura Kiken, Program on Integrative Medicine, UNC School of Medicine, CB \#7200, Chapel Hill, NC 27599. Phone: 919-966-8586. Fax: 919-843-5452. laura_kiken@med.unc.edu.

Publisher's Disclaimer: This is a PDF file of an unedited manuscript that has been accepted for publication. As a service to our customers we are providing this early version of the manuscript. The manuscript will undergo copyediting, typesetting, and review of the resulting proof before it is published in its final citable form. Please note that during the production process errors may be discovered which could affect the content, and all legal disclaimers that apply to the journal pertain.
} 
outcomes, raising questions about how mindfulness relates to more basic psychological processes. Though mindfulness might influence several facets of basic psychological processes, an important yet rather neglected aspect to investigate is thoughts that emphasize, or are weighted toward, positivity or negativity.

Individuals generally have and presumably need a mix of positive, negative, and neutral thoughts, but it is important to consider the degree to which they tend to emphasize the affective valence of incoming information with thoughts that are weighted toward positivity or negativity. Although the terms 'positive thinking' and 'negative thinking' are common shorthand for the notion that thoughts of a particular valence are relatively dominant compared to thoughts of the opposite valence or neutral thoughts, we primarily employ the terms emphasis on, and weighting toward, positivity or negativity for greater clarity. The valence emphasized in thoughts typically is consequential for psychological distress and well-being. A large body of psychological evidence implicates thoughts that emphasize negativity in stress and depression, whereas thoughts that emphasize positivity often, although not always, can benefit well-being (cf. Aspinwall \& Tedeschi, 2010; Beck, 2008). For example, from the standpoint of emotion regulation, thoughts that emphasize the valence of affective information (e.g., negatively weighted thoughts in response to negative emotions) can upregulate that affective state, increasing its intensity and duration (Watkins, 2008). This is one reason why rumination (i.e., repetitively thinking about an experience that has passed) on negative emotions tends to be more consistently problematic (e.g., promotes depression) than rumination on positive emotions (e.g., promotes positive affect that is beneficial to many populations; cf. Watkins, 2008).

Such tendencies toward negatively or positively weighted thoughts influence psychological health through other means, beyond emotion regulation, as well. For example, negatively biased cognitive styles (marked by dysfunctional beliefs and attitudes, biased information processing, and cognitive distortions; cf. Beck, 2008) predict negative life events, at least among depression-prone individuals (Safford, Alloy, Abramson, \& Crossfield, 2007). Pessimism predicts avoidance and less persistence in response to stressors and can lead to health-damaging behavior (Carver, Scheier, \& Segerstrom, 2010). Perhaps for these types of reasons, better psychological adjustment in nonclinical populations generally is marked by less negatively weighted thoughts, based on evidence from several studies using a simple thought-listing task (cf. Cacioppo, von Hippel, \& Ernst, 1997).

At the same time, several types of positively weighted thoughts appear to contribute to wellbeing. Optimism and benefit finding enhance coping processes (Aspinwall \& Tedeschi, 2010; Carver et al., 2010). Savoring, which in some forms is similar to rumination on positive affect but is not necessarily focused on past experience, can contribute to beneficial positive affect both in depressed (Hohn et al., 2013) and healthy individuals (Quoidbach, Berry, Hansenne, \& Mikolajczak, 2010). It should be noted that thoughts that emphasize positivity might promote dysfunction among individuals who are predisposed to bipolar disorder (Johnson, McKenzie, \& McMurrich, 2008), and there may be other cases in which positively weighted thoughts could have disadvantages (Crocker \& Park, 2004; Carver et al., 2010). Even with those caveats, there are clearer and more consistent risks to psychological 
health from thoughts emphasizing negativity compared to positivity, and the latter has potential for benefits.

Some theory and evidence suggest that mindfulness may differentially relate to thoughts emphasizing negativity or positivity. According to Buddhist-derived theories, mindfulness entails or enables a decentered awareness of thoughts that may reduce the tendency to be habitually swept away in and attached to certain streams of thought, regardless of whether they concern pleasant or unpleasant information (Gunaratana, 2002; Grabovac, Lau, \& Willett, 2011). It thus might be reasonable to hypothesize that more mindful individuals may be less mired in thoughts that emphasize valence in general, regardless of whether the valence is positive or negative. Alternatively, it also has been proposed that mindfulness may promote cognitive flexibility and choicefulness to use thoughts in more wholesome and adaptive ways (cf. Brown et al., 2007; Garland, Gaylord, \& Fredrickson, 2011). This (rather than habitual attachments) could promote fewer negatively weighted and more positively weighted thoughts, particularly within a larger milieu that encourages valence differences. Not only might Western lay psychology provide such a context, but at least some Buddhist teachings are relatively consistent with Western empirical psychology in that negative thoughts require particular care (i.e., by not dwelling on or being influenced by them) because they tend to manifest harm to the self or others more readily than neutral or positive thoughts (Groth-Marnat, 1992). Concomitantly, mindfulness often is taught (e.g., as in Mindfulness-Based Stress Reduction; Kabat-Zinn, 1990) in conjunction with practices and philosophies that prescribe intentional cultivation of certain positive thoughts. Examples include statements silently repeated in lovingkindness, or metta, meditation, such as "may all beings live with ease," and exercises that aim to increase awareness of pleasant experiences.

Preliminary empirical evidence suggests that mindfulness may affect the valence of thoughts, although much of this research was not designed to examine valence differences and has not adequately controlled for alternative explanations. Correlational studies suggest that trait mindfulness is inversely associated with tendencies toward negatively weighted thoughts including depressive rumination (cf. Keng et al., 2011), frequent and persistent dysfunctional thoughts (Frewen, Evans, Maraj, Dozois, \& Partridge, 2008), and negative cognitive styles that characterize depression and anxiety (Kiken \& Shook, 2012). Other correlational evidence suggests that trait mindfulness is positively associated with tendencies toward positively weighted thoughts, including optimism (cf. Brown et al., 2007), positive reappraisals (Garland et al., 2011), and self-enhancing positive illusions that may protect psychological health (Boatright \& McIntosh, 2008). One correlational study on mindfulness was designed to examine valence differences as a dimension of repetitive thinking, and found that several facets of trait mindfulness were associated with less negatively valenced and more positively valenced repetitive thought (Evans \& Segerstrom, 2011).

Still, these correlational studies have not adequately controlled for potential alternative explanations, such as affect. Affect is associated with trait mindfulness and can play a role in responses to measures of valenced cognition (e.g., individuals with more negative affect may be less mindful and also report more negative thoughts and fewer positive thoughts; cf. 
Fresco, Heimberg, Abramowitz, \& Bertram, 2006; Jislin-Goldberg, Tanay, \& Bernstein, 2012; Keng et al., 2011). Therefore, affect needs to be accounted for in a correlational design to determine whether trait mindfulness uniquely relates to thoughts that emphasize positive or negative valence. In addition, an experimental design would help to isolate mindfulness from other potential third variables that may relate to the valence of thoughts. For experimental designs, inducing mindfulness through meditation in the laboratory can provide a tightly controlled test of effects of a more mindful state than typical mental states. Although the "dose" of mindfulness may be modest, this method helps to isolate aspects of mindfulness from other constructs and potential experimental contaminants typically introduced in longer meditation trainings.

Although several studies have employed such induction methods, only two studies, to our knowledge, have done so to directly examine how mindfulness affects the valence of thoughts, or cognition more broadly. Alberts and Thewissen (2011) found that a brief mindful breathing meditation, compared to a control condition that did not meditate, reduced recall of negative words but not positive words on a memorization task. This effect was not due to differences in affect or total number of words recalled. However, an active, comparable control condition is ideal for eliminating potential confounds. Kiken and Shook (2011) compared a mindful breathing meditation to an active control condition, an unfocused attention exercise, and found that the mindful breathing condition subsequently reported more optimism but there was no difference for pessimism. In sum, these experiments found inconsistent effects for positive and negative cognitions, perhaps due to either different control conditions or different measures of cognition. Altogether, these experimental studies of mindfulness induced by meditation, along with correlational studies of trait mindfulness, introduce but do not sufficiently answer the question of whether mindfulness differentially affects thoughts that emphasize positivity and negativity.

More research is needed to directly examine mindfulness and thought valence, examining both positivity and negativity, while controlling for the role of affect and more generally attempting to isolate mindfulness from related constructs. The current research aimed to fill this gap in the literature through two studies that examined thoughts emphasizing positivity and negativity in response to affective information. The general hypothesis was that, after controlling for state affect (Study 1) and experimentally inducing mindfulness (Study 2), more mindful individuals would demonstrate less negatively weighted thoughts. We did not have a specific a priori hypothesis regarding mindfulness and positively weighted thoughts due to mixed theoretical perspectives and evidence in the literature, but we aimed to explore this potential relation with greater precision.

\subsection{Study 1}

The aim of Study 1 was to explore the relation between dispositional mindfulness and dispositional tendency to ruminate in response to affect. Rumination is a form of thought that emphasizes negativity or positivity (Watkins, 2008). Unlike previous studies on mindfulness, to determine the role of valence both positively and negatively valenced rumination were examined. Based on previous evidence, we hypothesized that more mindful individuals would demonstrate less negative rumination. Some previous correlational 
evidence suggests that mindfulness is associated with positive repetitive thought (Evans \& Segerstrom, 2011); however, because not all mindfulness theory and research consistently supports an association with positivity, we did not have an a priori hypothesis regarding positive rumination.

\subsection{Method}

2.1.1 Participants and Procedure-A convenience sample of 159 undergraduate students $\left(59 \%\right.$ female; $45 \%$ White; $M_{\text {age }}=20.26$ years, $\left.S D=4.00\right)$ took part in the study for extra course credit. This sample size was more than adequate to detect a medium effect (with $\alpha=.05$, power $=.80$, and 6 predictors), as determined using the program GPower (Faul, Erdfelder, Lang, \& Buchner, 2007). Several previous studies of associations between mindfulness and positive or negative cognition have reported medium to large effect sizes (e.g., Brown \& Ryan, 2003; Evans \& Segerstrom, 2011; Frewen et al., 2008; Garland et al., 2011; Kiken \& Shook, 2012).

Sessions were run in groups of at most eight participants. Upon arrival, participants were seated at individual computer cubicles and completed the measures described below, as well as a few unrelated measures that were part of a larger project. Upon completion, participants were debriefed, thanked, and dismissed.

\subsubsection{Measures}

Dispositional mindfulness: Two of the most widely used measures of dispositional mindfulness were employed given that there currently is debate over operationalizations of mindfulness. Although a subscale of the second measure shares some items with the first, they are not identical and it seemed prudent to allow for the possibility that they might produce distinct results.

The first measure was the Mindful Attention Awareness Scale (MAAS; Brown \& Ryan, 2003). The MAAS is a unidimensional self-report measure of present-moment oriented attention and awareness which inherently entails a quality of acceptance or receptivity. An example item is: "I find it difficult to stay focused on what's happening in the present." The 15 negatively worded items were scored on a scale from 1 (almost always) to 6 (almost never). Although the items at face value seem to concern the absence of mindfulness, several studies support the validity of this measure for assessing receptive attention to and awareness of present-moment experience; the scale also demonstrates sound psychometric properties in various populations (Brown \& Ryan, 2003; Brown, Ryan, Loverich, Biegel, \& West, 2011). Higher mean scores reflect higher mindfulness.

The second mindfulness measure was the Five Facet Mindfulness Questionnaire (FFMQ; Baer et al., 2006), which assesses multiple dimensions of mindfulness for expanded content validity, especially as reflected in clinical approaches to mindfulness. The FFMQ includes 39 items rated on a scale ranging from 1 (never or very rarely true) to 5 (very often or always true). These items cover five factors: nonreactivity to inner experience ( 7 items; e.g., "I perceive my feelings and emotions without having to react to them"); observing or attending to sensations, perceptions, thoughts, and feelings ( 8 items; e.g., "When I take a 
shower or a bath, I stay alert to the sensations of water on my body"); acting with awareness and concentration (8 items; e.g., "I am easily distracted," reverse-scored; five of the items in this subscale are from the 15-item MAAS); describing / labeling with words ( 8 items; e.g., "I can easily put my beliefs, opinions, and expectations into words"); and nonjudging of experience ( 8 items; e.g., "I tell myself that I shouldn't be feeling the way I'm feeling," reverse-scored). Item scores were totaled for each subscale, in line with the multidimensional approach of the measure. Higher scores reflect higher levels of each facet.

Rumination: Two self-report measures were used to assess negative rumination and positive rumination. The Ruminative Responses Scale-Short Form (RRS; Treynor, Gonzalez, \& Nolen-Hoeksema, 2003) measured negative rumination. The RRS is based on Nolen-Hoeksema's highly regarded Response Styles Theory (1991) of depressive rumination. Participants indicated how often they think in ways described by the items when they feel down, sad, or depressed on a scale from 1 (Almost never) to 4 (Almost always). The short form of the RRS consists of 10 items assessing two factors: brooding (5 items; e.g., "Think about a recent situation, wishing it had gone better") and reflection (5 items; e.g., "Analyze recent events to try to understand why you are depressed"), without items assessing depressive symptoms. Higher scores indicate greater negative rumination.

The Responses to Positive Affect questionnaire (RPA; Feldman, Joormann, \& Johnson, 2008) includes three subscales, two of which were used in this study because they assess positive rumination and were created to parallel Nolen-Hoeksema's construct of negative rumination. Participants indicated how often they think in ways described by the items when they feel happy, excited, or enthused on a scale ranging from 1 (Almost never) to 4 (Almost always), similar to the RRS. Nine items assess the two facets of positive rumination: a focus on emotion and somatic experiences ( 5 items; e.g., "Think about how you feel up to doing everything"), and a focus on self-related affirmations and goals (4 items; e.g., "Think 'I am living up to my potential"'). Higher scores indicate greater positive rumination.

State affect: State affect was assessed by the Positive and Negative Affect Schedule (PANAS; Watson, Clark, \& Tellegen, 1988) to potentially account and control for its relation to the constructs of interest. Controlling for state affect was important in order to discern the extent to which dispositional mindfulness uniquely accounted for variation in trait rumination. It likewise helped to address a related potential source of measurement error, in that participants previously have been found to endorse positive or negative statements depending on whether they currently are in a positive or negative mood, respectively (e.g., Fresco, Heimberg, Abramowitz, \& Bertram, 2006). The PANAS is a commonly used self-report measure comprised of two subscales that assess two global dimensions of affect, positive and negative. Participants rate each of 20 adjectives (e.g., enthusiastic, distressed) using a scale ranging from 1 (very slightly or not at all) to 5 (very much), to indicate the extent to which they are currently experiencing the descriptor. Scores for each subscale were totaled, with higher scores indicating higher positive or negative affect. 


\subsection{Results}

Means, standard deviations, Cronbach's alphas, and inter-correlations for all Study 1 measures are presented in Table 1.

2.2.1 Did trait mindfulness predict negative rumination?-Replicating past research, the MAAS and several subscales of the FFMQ were inversely associated with the RRS total and subscale scores $(r \mathrm{~s}=-.17--.56)$. Because negative state affect also was associated with mindfulness $(r \mathrm{~s}=-.17--.41)$ and the RRS scores $(r \mathrm{~s}=.25-.38)$, two hierarchical regression models were used to examine if each of the mindfulness measures significantly predicted negative rumination above and beyond the role of negative state affect. In both models, negative state affect was entered as a predictor in the first step, and the mindfulness measure was entered as a predictor in the second step. We also added a third step to the models that included an interaction term created from the product of meancentered scores (cf. Aiken \& West, 1991) on the mindfulness (MAAS in Model 1; FFMQ subscales in Model 2) and negative affect scales. This tested whether the relation between negative state affectand negative rumination depended on mindfulness. Total RRS scores were used as the criterion variable in both models given that the mindfulness measures generally correlated with both RRS subscales.

Full results for both Model 1 and Model 2 are shown in Table 2. In Model 1, the MAAS was a significant predictor of negative rumination above and beyond the role of negative state affect. The interaction between the MAAS and negative affect was not significant. In Model 2, all FFMQ subscales were entered as predictors in the second step. After accounting for negative affect, only the Nonjudging subscale independently predicted less negative rumination. None of the interactions between the FFMQ subscales and negative affect were significant.

2.2.2 Did trait mindfulness predict positive rumination?-The MAAS and the FFMQ subscales all positively correlated with total RPA scores $(r s=.17-.27)$; additional positive correlations between the mindfulness measures and one or both of the RPA subscales were observed with general support for relations with both subscales $(r \mathrm{~s}=.18-$. $28)$. Because positive state affect also was associated with mindfulness $(r s=.18-.35)$ and the RPA scores $(r s=.38-.47)$, two hierarchical regression models were used to examine if each of the mindfulness measures significantly predicted positive rumination above and beyond the role of positive state affect, similar to the analyses for negative rumination. In both models, positive state affect was entered as a predictor in the first step, and the mindfulness measure was entered as a predictor in the second step. As with negative rumination, we then added a third step to the models to test interactions between the mindfulness (MAAS in Model 3; FFMQ subscales in Model 4) and positive affect scales. That is, we tested whether a relation between positive state affect and positive rumination depended on mindfulness. As with the RRS, total RPA scores were used as the criterion variable in both models given that the mindfulness measures generally correlated with both RPA subscales. 
Full results for both Model 3 and Model 4 are shown in Table 2. In Model 3, the MAAS did not significantly predict positive rumination above and beyond the role of positive state affect, and the interaction between the MAAS and positive affect was not significant. In Model 4, the FFMQ subscales were entered as predictors in the second step. After accounting for positive state affect, none of the FFMQ subscales significantly predicted positive rumination, although the Act with Awareness subscale approached significance. Again, none of the interactions between the FFMQ subscales and positive affect were significant.

\subsection{Discussion}

Study 1 examined dispositional mindfulness in relation to dispositional positive and negative rumination, to examine a form of thought that emphasizes positive or negative valence. The results replicated previous findings that trait mindfulness (both the MAAS and the FFMQ Nonjudging subscale) predicted less negative rumination. This relation was observable above and beyond the role of negative state affect and not moderated by negative state affect, supporting that mindfulness itself plays a consistent role in negative rumination. The MAAS and FFMQ Nonjudging subscale represent receptive present-moment attention and awareness and a nonjudgmental stance, respectively, which are central features of mindfulness according to many scholars (at least as characterized in the Western, secular literature, e.g., Bishop et al., 2004; Brown \& Ryan, 2003; Kabat-Zinn, 1990).

Importantly, the results also demonstrated that a bivariate positive relation between mindfulness and positive rumination was not significant after accounting for the role of positive state affect. This finding has two important implications. First, the results do not support that mindfulness is inversely associated with all rumination regardless of valence. Second, the results suggest that apparent positive associations between mindfulness and positive thinking (e.g., Evans \& Segerstrom, 2011) may largely be due to shared variance with positive state affect. Positive state affect may reflect either dispositional affective tendencies or random positive affect; the current findings do not disentangle these. Either way, the results do not provide strong support for a distinct role of mindfulness in positive rumination, unlike the results for negative rumination.

Overall, the findings from Study 1 suggest that mindfulness is associated with less negatively weighted thoughts, but is not directly related to positively weighted thoughts. To examine these relations with tighter control and a basis for directionality, we conducted an experiment in Study 2.

\subsection{Study 2}

Study 2 used a randomized, controlled experiment to examine the effects of a mindfulness induction, compared to a contrasting and typical mental state (i.e., unfocused attention), on positively or negatively weighted thoughts in response to affective stimuli. To assess on-line cognition, we used a standard thought listing procedure and visual stimuli that reliably produce positive and negative affect. 


\subsection{Method}

3.1.1 Participants-A convenience sample of 102 undergraduate students (different from those in Study 1) participated in the study for course credit (65\% female; $49 \%$ White; $M_{\text {age }}$ $=21.00$ years, $S D=3.73$ ). This sample size was adequate to detect a medium to large effect (with $a=.05$ and power $=.80$ ), as determined using GPower (Faul et al., 2007). As noted for Study 1, such effect sizes have been reported in several studies of associations between mindfulness and valenced thought (including Study 1 in the present research). Additionally, a medium-large effect was reported in Alberts and Thewissen's (2011) mindfulness induction study, which was similar to the current research in that it measured cognition in response to affective stimuli. The majority (75\%) of our sample had no previous experience with mindfulness meditation. To conceal the hypothesis, the study was described as a test of effects of mental exercises.

\subsubsection{Manipulation and Measures}

Mindfulness manipulation: For efficiency and control, mindfulness was manipulated using a laboratory-based induction that centered on the present-moment attention and nonjudgmental qualities of mindfulness, given that these two aspects of mindfulness are largely agreed upon by Western scholars and were related to rumination in Study 1. All participants listened to a standardized 10-minute audio recording. The instructions for each condition, which were similar to those used in other mindfulness induction studies (e.g., Arch \& Craske, 2006), have been described and pilot tested in previous research where they produced the expected differences in state mindfulness (Kiken \& Shook, 2011). Briefly, participants in the mindfulness condition received instruction in a mindful breathing meditation, attending to the sensations of each breath in and out to anchor their attention in their present experience. They also were instructed to acknowledge momentary thoughts and feelings as such, being aware of where the mind is with curiosity but not judgment, as part of the process of gently returning one's attention to present experience. Participants in the control condition received instruction to let their minds wander freely, letting themselves take the time to think through things without focusing on anything in particular. The control condition instructions were designed to mirror the length and pacing of the mindful breathing instructions and were narrated by the same voice. The manipulations were carefully designed to hold constant every aspect except the actual content of the instructions. Because these manipulations were pilot tested previously, we did not measure state mindfulness afterward to avoid potential demand effects.

Thought valence: To assess the valence of thoughts in response to positive and negative affective images, a common thought listing procedure (Cacioppo \& Petty, 1981; Cacioppo et al., 1997) was used while presenting eight different images from the International Affective Picture System (IAPS; Lang et al., 2005). Four of the images were positive (e.g., a man and a woman with bicycles, leaning toward each other and smiling in the sunshine), and the other four images were negative (e.g., a dead, dirty cat in the middle of a road near a moving tire). IAPS images were selected as stimuli because they have been normatively rated for valence and used in similar studies previously. However, to ensure that basic affective responses to the IAPS images were not affected by the mindfulness induction, while viewing each image participants first rated their affective state on a single item scale (Wolpe, 1990) 
from -50 (the most negative/unpleasant emotional state) to +50 (the most positive/pleasant emotional state) as in Arch and Craske (2006).

Then, while continuing to view the image for 20 additional seconds, participants were asked to "write down all of the thoughts you have now, advancing to a new screen for each thought by pressing 'enter' after each thought. Simply write down whatever comes to mind." The entire image viewing/rating and thought listing procedure lasted approximately three to four minutes.

The thought listing technique uses an open-ended response format to obtain and categorize participants' reported thoughts based on dimensions of interest, in this case valence. The first two images (one positive, one negative) served as practice; the remaining six images were coded for analysis. Two raters, who were blinded to experimental condition, coded the number and valence (positive, negative, or neither) of thoughts $(\mathrm{Ks}>.70)$, with discrepancies resolved by the first author. To determine whether participants' thoughts emphasized a particular valence, or no valence, in response to the images, the proportions of positive (e.g., "fun," "happy”), negative (e.g., "horrible," "depressing”), and non-valenced (e.g., "bicycle," "red") thoughts, out of the total number of thoughts, were calculated for each image. Then, these proportions were averaged for the positive images and the negative images. Higher numbers thus represent higher average percentages of each type of thought (positive, negative, or non-valenced) in response to positive images and negative images.

Dispositional mindfulness: The MAAS was included to ensure that the conditions did not differ on their levels of dispositional mindfulness. We selected this measure for its relative brevity and associations with related constructs of interest in Study 1. The MAAS also has demonstrated temporal stability (Brown \& Ryan, 2003) and explicitly instructs participants to reflect on their everyday experience, so it was unlikely to be affected by the study procedures.

3.1.3 Procedure-As in Study 1, sessions were run in groups of at most eight participants who were seated at individual computer cubicles. In each session, all participants were assigned to the same experimental condition to maintain a consistent environment and avoid potential distractions. Experimenters were blinded to condition assignment, as they simply entered a number " 1 " or " 2 " into the computer program and could not hear the audio recordings. Participants listened through individual headphones to the audio recording that served as the manipulation. The manipulation was immediately followed by the presentation of the IAPS images with an affect scale and thought listing for each image. Then, after completing additional, unrelated measures for a separate line of investigation (for approximately 10-15 minutes), participants completed the MAAS. Finally, participants were debriefed, thanked, and dismissed.

\subsection{Results}

\subsubsection{Preliminary Analyses}

Was randomization successful?: A $t$-test determined that the experimental groups did not differ significantly on trait levels of mindfulness, $t(100)=-.16, p=.87$. The groups also did 
not differ significantly in their previous experience with mindfulness meditation, $X^{2}(5$, $N=102)=4.70, p=.45$.

Did the IAPS images produce intended affective responses?: A 2 (experimental condition) $\times 2$ (image valence) mixed factorial ANOVA was used to examine participants' affective responses to the IAPS images and whether these varied by experimental condition. Experimental condition was entered as a between-subjects variable, and image valence was entered as a within-subjects variable. There was a main effect of image valence, Wilks' Lambda $=.12, F(1,100)=766.13, p<.001, \eta_{\text {partial }}^{2}=.86$, such that participants reported more negative affective responses to negative images $(M=-29.03, S D=17.50)$ and more positive affective responses to positive images $(M=30.20, S D=12.10)$. Neither the main effect of condition nor the interaction of image valence and condition were significant ( $p \mathrm{~s}$ $>$.50). Thus, the IAPS images produced the expected affective responses, which were not affected by the manipulation.

\subsubsection{Main Analyses}

Did mindfulness meditation affect the valence of thoughts in response to affective images?: To test for differences between experimental conditions on the proportions of negative, non-valenced, and positive thoughts in response to the positive and negative images, we first conducted a 2 (experimental condition) $\times 3$ (thought valence) $\times 2$ (image valence) mixed factorial ANOVA. Condition was entered as a between-subjects variable. Thought valence and image valence were entered as within-subjects variables. The proportions of positive, negative, and non-valenced thoughts in response to positive and negative images, by condition, are shown in Figure 1. For this analysis, Box's M statistic could not be computed due to a singularity issue in the covariance matrix, largely due to very low proportions of thoughts of opposite valence to the image valence (e.g., negative thoughts in response to positive images). Thus, results from this analysis warrant caution.

First, there was a significant interaction between thought valence and image valence, Wilks' Lambda $=.12, F(2,95)=358.66, p<.001, \eta^{2}$ partial $=.88$, with participants' thoughts emphasizing the valence of the image (see Figure 1). There was also a marginally significant two-way interaction between condition and thought valence, Wilks' Lambda $=.94, F(2,95)$ $=2.80, p=.07, \eta^{2}$ partial $=.06$. Both of these interactions were qualified by a marginally significant three-way interaction between condition, thought valence, and image valence, Wilks' Lambda $=.94, F(2,95)=2.95, p=.06, \eta^{2}$ partial $=.06$. None of the other main effects or interactions were statistically significant $(p s>.11)$. To explore these interactions and given that these statistics may have been unreliable, we conducted three separate mixed factorial ANOVAs that met the assumption that the within-group covariance matrices were equal as tested by Box's M.

Did mindfulness meditation affect negatively valenced thoughts?: A 2 (condition) $\times 2$ (image valence) mixed factorial ANOVA tested whether the proportion of negatively valenced thoughts differed by condition (between-subjects variable) and whether this depended on image valence (within-subjects variable). There was a significant main effect of image valence, Wilks' Lambda $=.18, F(1,96)=434.71, p<.001, \eta^{2}$ partial $=.82$, with 
proportionately more negative thoughts in response to negative images $(M=.56, S D=.24)$ than positive images $(M=.06, S D=.09)$. There also was a significant main effect of condition, $F(1,96)=5.27, p=.02, \eta^{2}$ partial $=.05$, with the mindfulness condition (estimated $M=.28, S E=.02$ ) listing proportionately fewer negative thoughts than the control condition (estimated $M=.34, S E=.02$ ). However, these main effects were qualified by a significant interaction between condition and image valence, Wilks' Lambda $=.94, F(1,96)=5.67, p$ $=.02, \eta_{\text {partial }}^{2}=.06$. The mindfulness condition listed proportionately fewer negative thoughts in response to negative images $(M=.50, S D=.25)$ than the control condition $(M$ $=.62, S D=.22), p=.01$. The proportion of negative thoughts in response to positive images did not differ between conditions, $p=.73$. There may have been a floor effect for positive images, however, given that the proportion of negative thoughts in response to positive images was very low for both conditions (mindfulness: $M=.06, S D=.08$; control: $M=.07$, $S D=.10)$.

Did mindfulness meditation affect positively valenced thoughts?: A similar 2 (condition) $\times 2$ (image valence) mixed factorial ANOVA tested whether the proportion of positively valenced thoughts differed by condition and whether this depended on image valence. There was a significant main effect of image valence, Wilks' Lambda $=.15, F(1,96)=549.34, p$ $<.001, \eta_{\text {partial }}^{2}=.85$, with participants listing proportionately more positive thoughts in response to positive images $(M=.62, S D=.24)$ than negative images $(M=.06, S D=.10)$. There was neither a main effect of condition, $F(1,96)=1.03, p=.31$, nor an interaction between condition and image valence, Wilks' Lambda $=1.00, F(1,96)=.13, p=.72$.

Did mindfulness meditation affect non-valenced thoughts?: A final 2 (condition) $\times 2$ (image valence) mixed factorial ANOVA tested whether the proportion of non-valenced thoughts differed by condition and whether this depended on image valence. There was a significant main effect of image valence, Wilks' Lambda $=.95, F(1,96)=4.65, p=.03$, $\eta_{\text {partial }}^{2}=.05$, with participants listing proportionately more non-valenced thoughts in response to negative images $(M=.37, S D=.26)$ than positive images $(M=.33, S D=.25)$. There also was a significant main effect of condition, $F(1,96)=4.60, p=.04$, with the mindfulness condition (estimated $M=.40, S E=.03$ ) listing proportionately more nonvalenced thoughts than the control condition (estimated $M=.31, S E=.03$ ). The interaction between condition and image valence was not significant, Wilks' Lambda $=.97, F(1,96)=$ $3.23, p=.08$.

3.2.3 Post-hoc analysis-With the differences between the mindfulness and control conditions on their proportions of negative and non-valenced thoughts, it was conceivable that one condition might have listed fewer thoughts. However, a 2 (condition) $\times 2$ (image valence) mixed factorial ANOVA confirmed that there were no differences between conditions on the average number of thoughts listed overall $(p=.46)$ or for the interaction between condition and image valence $(p=.44)$.

\subsection{Discussion}

Study 2 used a randomized experiment to test the effect of a mindfulness induction on the valence of thoughts in response to positive and negative affective images. The proportion of 
positively valenced thoughts was not affected by the mindfulness induction, whereas the proportions of negatively valenced and non-valenced thoughts were. Individuals induced to be more mindful listed proportionately fewer negative thoughts and proportionately more non-valenced thoughts. Whether this overall pattern was specific to negative images was somewhat unclear, for two reasons. On one hand, the interaction between condition and image valence for non-valenced thoughts was nonsignificant, although the pattern was in the same direction as for negative thoughts (i.e., a greater between-condition difference for negative images than for positive images). On the other hand, the significant interaction between condition and image valence for negative thoughts could have been due to very low levels of negative thoughts in both conditions for positive images. Either way, the evidence supports a general conclusion that mindfulness meditation resulted in proportionately fewer negative and more non-valenced thoughts, but did not affect positive thoughts. Importantly, these between-condition differences were not due to affect, as the conditions did not differ on affective responses to the images. These effects were also not simply due to one condition having more or fewer thoughts than the other condition, as the conditions did not differ in number of thoughts listed. Thus, the results indicate that the mindfulness induction affected thought valence.

It is worth considering, as an alternative explanation, whether the control condition instructions (unfocused attention) may have increased the proportion of negative thoughts and decreased the proportion of non-valenced thoughts. It seems more likely that the observed effects were driven by the mindfulness induction, for several reasons. First, the proportion of negative thoughts in response to positive images remained low in the control condition, which would not have been the case if the control exercise increased negative thoughts overall. Second, in the control condition, the proportion of negative thoughts in response to negative images was comparable to the proportion of positive thoughts in response to positive images, suggesting a typical pattern of thoughts emphasizing the valence of the image. It seems less likely that the unique pattern of thoughts in the mindfulness condition represents typical cognition. Finally, an effect of mindfulness on negatively valenced cognition is supported by previous evidence (e.g., Alberts \& Thewissen, 2011).

\subsection{General Discussion}

The current research utilized two studies to examine the relation between mindfulness and thoughts that emphasize positivity or negativity, to investigate potential differences by valence. Together, the results from Study 1 (correlational) and Study 2 (experimental) suggest that mindfulness may reduce thoughts that emphasize negativity, as hypothesized. The results also suggest that mindfulness does not reduce thoughts that emphasize positivity. Therefore, it cannot be concluded that mindfulness reduces all thoughts emphasizing valence, in general. Mindfulness was specifically related (inversely) to negatively weighted thoughts. These results were based on study designs that isolated the construct of mindfulness from state affect (Study 1) and other potential third variables (Study 2). Further, the randomized, controlled experimental design in Study 2 demonstrated causality. The Study 2 results also suggest that when being mindful, individuals may have more nonvalenced thought responses to affective information instead of negative thoughts. 
These findings help to clarify the relation between mindfulness and basic psychological processes, positively and negatively weighted thinking, that typically are consequential for psychological distress and well-being (Aspinwall \& Tedeschi, 2010; Beck, 2008). Our results are consistent with a growing evidence base suggesting that mindfulness may reduce cognitive emphasis on negativity, including various types of negative cognitions that may contribute to distress and dysfunction (Alberts \& Thewissen, 2011; Frewen et al., 2008; Kiken \& Shook, 2012; Raes \& Williams, 2010). Some previous studies have been framed in terms of mindfulness influencing other aspects of thinking related to cognitive control, such as the tendency to uncontrollably ruminate versus "let go," with less attention to the role of valence. It is important to consider that such cognitive control may be employed primarily to prevent or reduce emphasis on negative information. It also is important to note that the present research does not indicate or suggest that mindfulness is associated with low levels of negative thoughts, but thoughts weighted toward, or emphasizing, negativity in response to affective information were attenuated. In future research, investigators should consider reasons why more mindful individuals' thoughts might specifically de-emphasize negativity. For instance, this phenomenon may relate to knowledge or beliefs about potential emotional and behavioral consequences of emphasizing negativity, which might be acted on through greater cognitive control.

The current studies also provide consistent evidence that mindfulness, isolated from correlates, is unrelated to thoughts that emphasize positivity. Some previous theory and research has linked mindfulness and at least certain forms of thought emphasizing positivity (e.g., Boatright \& McIntosh, 2008; Evans \& Segerstrom, 2011; Kiken \& Shook, 2011). It seems likely that associations in correlational and intervention-based studies may be due to: (a) common correlates of mindfulness (e.g., positive affect, as in Study 1 here, and possibly also cognitive flexibility; cf. Garland et al., 2011), or (b) related but potentially confounding practices that are included in multi-component mindfulness interventions (e.g., lovingkindness meditation). These alternative explanations should be considered in future research on mindfulness and positively weighted thoughts. However, they do not necessarily explain the inconsistent findings of randomized, controlled studies using mindfulness inductions. The findings of the present research align with those of Alberts and Thewissen (2011) in that the mindfulness induction did not affect positively valenced thoughts in response to affective stimuli. The other previous mindfulness induction study that assessed a type of thinking that emphasizes valence showed an increase in positively weighted thought (Kiken \& Shook, 2011), using a self-report measure of optimistic expectations. The more subjective or dispositional nature of this measure may account for the discrepant finding. Additionally, it is possible that mindfulness facilitates only some types of thoughts that emphasize positivity. These particulars could be investigated in future research.

Limitations of this research include the use of undergraduate convenience samples, which may not generalize to clinical or other populations for which thoughts that emphasize negativity or positivity may be particularly relevant. In addition, Study 1 used only selfreport measures, which are subject to biases, although accounting for the influence of state affect was a relative strength. Further, two widely used measures of trait mindfulness were employed and showed similar results. Considering operationalizations of mindfulness, however, it is possible that both self-report measures of trait mindfulness and the mindful 
breathing manipulation represent certain Western conceptualizations of mindfulness but not necessarily the ideals of mindfulness according to some Buddhist spiritual philosophies or meditation traditions. Different operationalizations of mindfulness might produce different results.

Study 2 had other limitations to consider as well. It is possible that brief mindfulness meditation only has a small effect on positively weighted thinking as we assessed it, and our sample size was not large enough to detect small effects. However, this still would support a stronger effect of mindfulness on negatively weighted thinking. Another consideration when interpreting the results from Study 2 is that thoughts categorized as non-valenced may have been ambivalent or unclear. Further limitations of the thought listing include that participants' subjective evaluations might differ from typical categorizations of valence (e.g., "scary" could be appealing to some); in addition, we did not distinguish thoughts based on extremity (e.g., both "inconvenience" and "disaster" were simply categorized as negative). It also is possible that the thought listing paradigm taps different underlying aspects of cognition than the rumination measures in Study 1. Nonetheless, the thought listing paradigm has a strong history of use (cf. Cacioppo et al., 1997) and was well-suited to the study of basic valence differences in thought responses to affective information.

Despite these limitations, the current research contributes to a growing literature on mindfulness and basic psychological processes that may underlie benefits for psychological health. Less negatively weighted thoughts, as assessed by the measures used in this research, appear to be protective for psychological health (cf. Cacioppo et al., 1997; Watkins, 2008). Thus, the current findings align with the notion that mindfulness may prevent or reduce negative psychological outcomes (e.g., distress) partly because it attenuates potentially detrimental cognitive emphasis on negativity. The current work also suggests that mindfulness neither promotes nor precludes thoughts that emphasize positivity, and calls for more precise research on the nuances of mindfulness and positive cognition and affect.

\section{Acknowledgments}

This work was partially supported by the National Center for Complementary and Alternative Medicine of the National Institutes of Health under award number T32AT003378. The content is solely the responsibility of the authors and does not necessarily represent the official views of the National Institutes of Health.

This research was partly conducted at Virginia Commonwealth University. Thank you to Lauren Moore, Paul Plonski, and Michael Nargi for their research assistance.

\section{References}

Aiken, LS.; West, SG. Multiple regression: Testing and interpreting interactions. Newbury Park, CA: SAGE; 1991.

Alberts HJEM, Thewissen R. The effect of a brief mindfulness intervention on memory for positively and negatively valenced stimuli. Mindfulness. 2011; 2:73-77. [PubMed: 21660085]

Arch JJ, Craske MG. Mechanisms of mindfulness: Emotion regulation following a focused breathing induction. Behavior Research and Therapy. 2006; 44(12):1849-1858.

Aspinwall LG, Tedeschi RG. The value of positive psychology for health psychology: Progress and pitfalls in examining the relation of positive phenomena to health. Annals of Behavioral Medicine. 2010; 39:4-15. [PubMed: 20091429] 
Baer RA, Smith GT, Hopkins J, Krietemeyer J, Toney L. Using self-report assessment methods to explore facets of mindfulness. Assessment. 2006; 13(1):27-45. [PubMed: 16443717]

Beck AT. The evolution of the cognitive model of depression and its neurobiological correlates. American Journal of Psychiatry. 2008; 165:969-977. [PubMed: 18628348]

Bishop SR, Lau M, Shapiro S, Carlson L, Anderson ND, Carmody J, Devins G. Mindfulness: A proposed operational definition. Clinical Psychology: Science and Practice. 2004; 11(3):230-241.

Boatright RA, McIntosh WD. The relationship between mindfulness and self-promoting illusions. Mental Health, Religion, \& Culture. 2008; 11:561-566.

Brown KW, Ryan RM. The benefits of being present: Mindfulness and its role in psychological wellbeing. Journal of Personality and Social Psychology. 2003; 84(4):822-848. [PubMed: 12703651]

Brown KW, Ryan RM, Creswell JD. Mindfulness: Theoretical foundations and evidence for its salutary effects. Psychological Inquiry. 2007; 18(4):211-237.

Brown KW, Ryan RM, Loverich TM, Biegel GM, West AM. Out of the armchair and into the streets: Measuring mindfulness advances knowledge and improves interventions: Reply to Grossman. 2011

Carver CS, Scheier MF, Segerstrom SC. Optimism. Clinical Psychology Review. 2010; 30:879-889. [PubMed: 20170998]

Cacioppo, JT.; Petty, RE. Social psychological procedures for cognitive response assessment: The thought-listing technique. In: Merluzzi, TV.; Glass, CR.; Genest, M., editors. Cognitive Assessment. New York: Guilford Press; 1981. p. 309-342.

Cacioppo JT, von Hippel W, Ernst JM. Mapping cognitive structures and processes through verbal content: The thought-listing technique. Journal of Consulting and Clinical Psychology. 1997; 65:928-940. [PubMed: 9420354]

Crocker J, Park LE. The costly pursuit of self-esteem. Psychological Bulletin. 2004; 130:392-414. [PubMed: 15122925]

Evans DR, Segerstrom SC. Why do mindful people worry less? Cognitive Therapy and Research. 2011; 35(6):505-510.

Faul F, Erdfelder E, Lang A-G, Buchner A. G*Power 3: A flexible statistical power analysis program for the social, behavioral, and biomedical sciences. Behavior Research Methods. 2007; 39:175191. [PubMed: 17695343]

Feldman GC, Joormann J, Johnson SL. Responses to positive affect: A self-report measure of rumination and dampening. Cognitive Therapy and Research. 2008; 32(4):507-525. [PubMed: 20360998]

Fresco DM, Heimberg RG, Abramowitz A, Bertram TL. The effect of a negative mood priming challenge on dysfunctional attitudes, explanatory style, and explanatory flexibility. British Journal of Clinical Psychology. 2006; 45:167-183. [PubMed: 16719978]

Frewen PA, Evans EM, Maraj N, Dozois DJA, Partridge K. Letting go: Mindfulness and negative automatic thinking. Cognitive Therapy and Research. 2008; 32(6):758-774.

Garland EL, Gaylord SA, Fredrickson BL. Positive reappraisal mediates the stress-reductive effects of mindfulness: An upward spiral process. Mindfulness. 2011; 2:59-67.

Grabovac AD, Lau MA, Willett BR. Mechanisms of mindfulness: A Buddhist psychological model. Mindfulness. 2011; 2:154-166.

Groth-Marnat, G. Buddhism and mental health: A comparative analysis. In: Schumaker, JF., editor. Religion and Mental Health. New York: Oxford University Press; 1992. p. 270-280.

Gunaratana, BH. Mindfulness in plain English. Boston: Wisdom Publications; 2002.

Höhn P, Menne-Lothmann C, Peeters F, Nicolson NA, Jacobs N, et al. Moment-to-moment transfer of positive emotions in daily life predicts future course of depression in both general population and patient samples. PLoS ONE. 2013; 8(9):e75655. [PubMed: 24086602]

Jislin-Goldberg T, Tanay G, Bernstein A. Mindfulness and positive affect: cross-sectional, prospective intervention, and real-time relations. The Journal of Positive Psychology. 2012; 7:349-361.

Johnson SL, McKenzie G, McMurrich S. Ruminative responses to negative and positive affect among students diagnosed with bipolar disorder and major depressive disorder. Cognitive Therapy and Research. 2008; 32:702-713. [PubMed: 20360996] 
Kabat-Zinn, J. Full catastrophe living: Using the wisdom of your mind to face stress, pain and illness. New York: Dell; 1990.

Keng SL, Smoski MJ, Robins CJ. Effects of mindfulness on psychological health: A review of empirical studies. Clinical psychology review. 2011; 31(6):1041-1056. [PubMed: 21802619]

Khoury B, Lecomte T, Fortin G, Masse M, Therien P, Bouchard V, ... Hofmann SG. MindfulnessBased therapy: A comprehensive meta-analysis. Clinical Psychology Review. 2013; 33(6):763771. [PubMed: 23796855]

Kiken LG, Shook NJ. Looking up: Mindfulness increases positive judgments and reduces negativity bias. Social Psychological and Personality Science. 2011; 2(4):425-431.

Kiken LG, Shook NJ. Mindfulness and emotional distress: The role of negatively biased cognition. Personality and Individual Differences. 2012; 52:329-333.

Lang, PJ.; Bradley, MM.; Cuthbert, BN. International Affective Picture System (IAPS): Technical manual and affective ratings. Gainesville: University of Florida, Center for Research in Psychophysiology; 2005.

Nolen-Hoeksema S. Responses to depression and their effects on the duration of depressive episodes. Journal of Abnormal Psychology. 1991; 100:569-582. [PubMed: 1757671]

Quoidbach J, Berry EV, Hansenne M, Mikolajczak M. Positive emotion regulation and well-being: Comparing the impact of eight savoring and dampening strategies. Personality and Individual Differences. 2010; 49:368-373.

Raes F, Williams JMG. The relationship between mindfulness and uncontrollability of ruminative thinking. Mindfulness. 2010; 1(4):199-203.

Safford SM, Alloy LB, Abramson LY, Crossfield AG. Negative cognitive style as a predictor of negative life events in depression-prone individuals: A test of the stress generation hypothesis. Journal of Affective Disorders. 2007; 99:147-154. [PubMed: 17030064]

Treynor W, Gonzalez R, Nolen-Hoeksema S. Rumination Reconsidered: A Psychometric Analysis. Cognitive Therapy and Research. 2003; 27(3):247-259.

Watkins ER. Constructive and unconstructive repetitive thought. Psychological Bulletin. 2008; 134:163-206. [PubMed: 18298268]

Watson D, Clark LA, Tellegen A. Development and validation of brief measures of positive and negative affect: The PANAS scales. Journal of Personality and Social Psychology. 1988; 54(6): 1063-1070. [PubMed: 3397865]

Wolpe, J. The practice of behavior therapy (Rev. ed.). New York: Pergamon Press; 1990. 


\section{Highlights}

- Mindfulness was examined in relation to positively and negatively weighted thoughts.

- Study 1 examined trait mindfulness in relation to positive and negative rumination.

- Study 2 manipulated mindfulness, then assessed thoughts listed while viewing images.

- Mindfulness did not uniquely relate to emphasis on positive thoughts.

- Both studies suggested that mindfulness may attenuate emphasis on negative thoughts. 


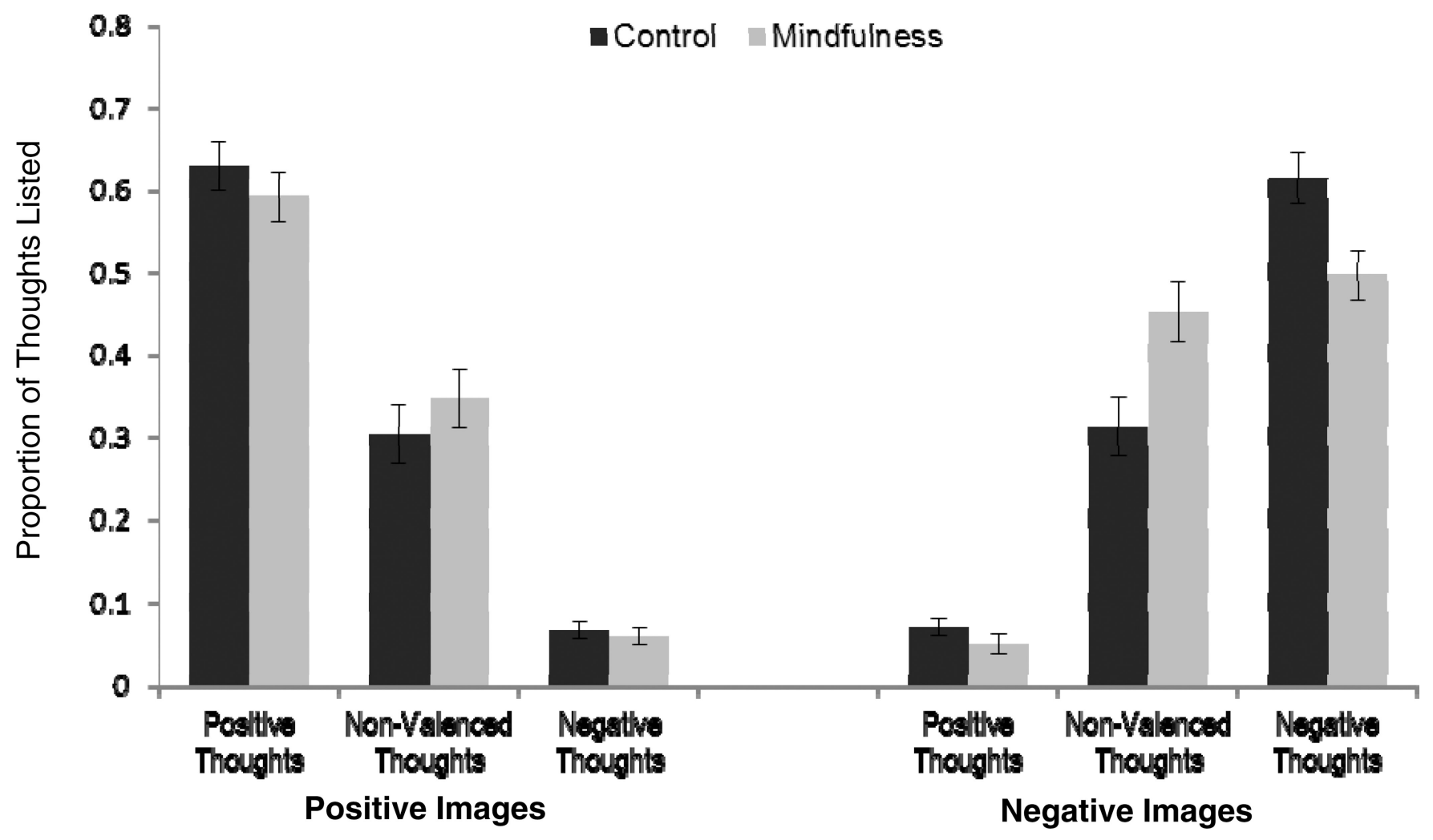

Figure 1.

Mean proportions of positive, non-valenced, and negative thoughts, by experimental condition, in response to positive and negative affective images. Bars indicate $+/-1 S E$. 


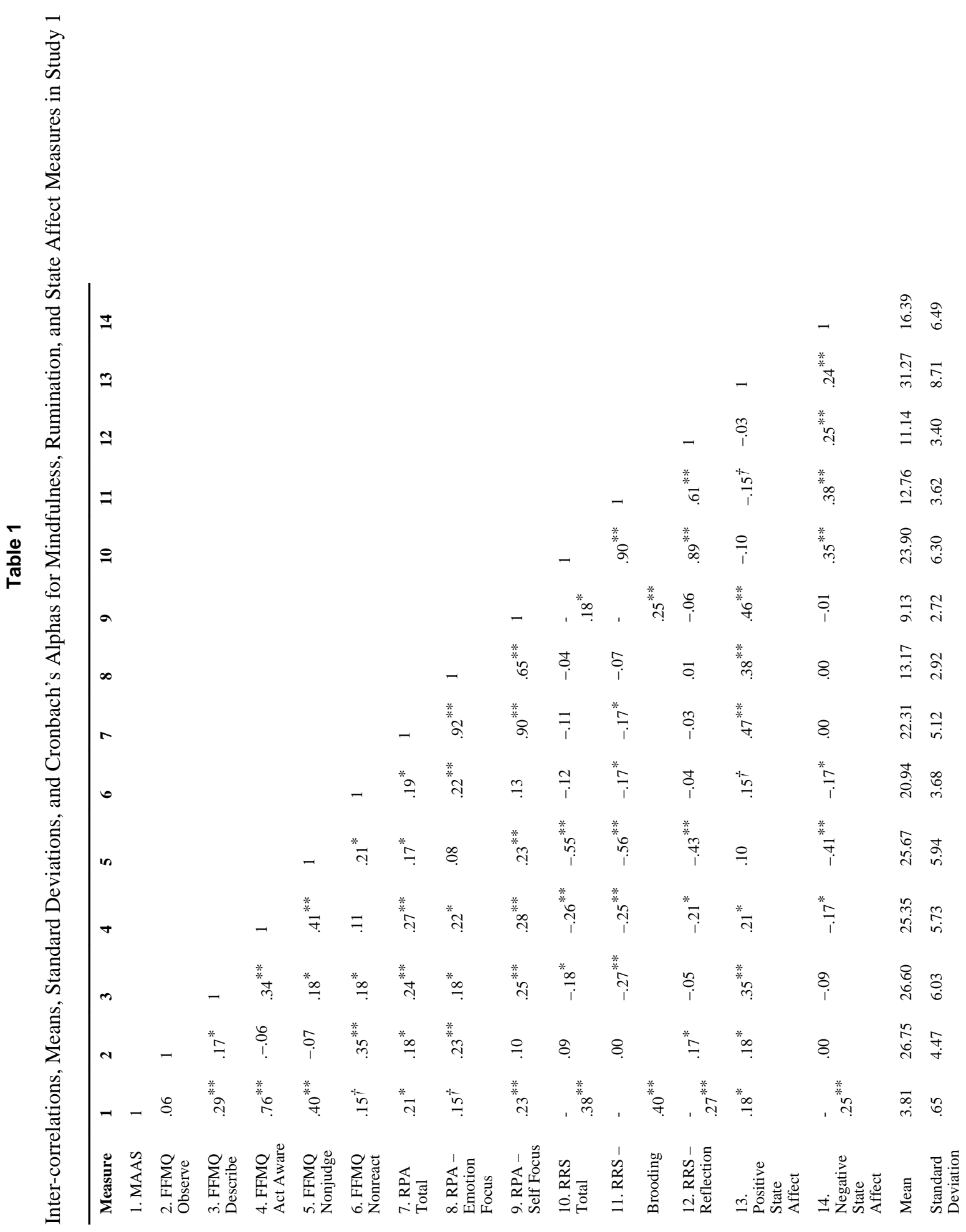




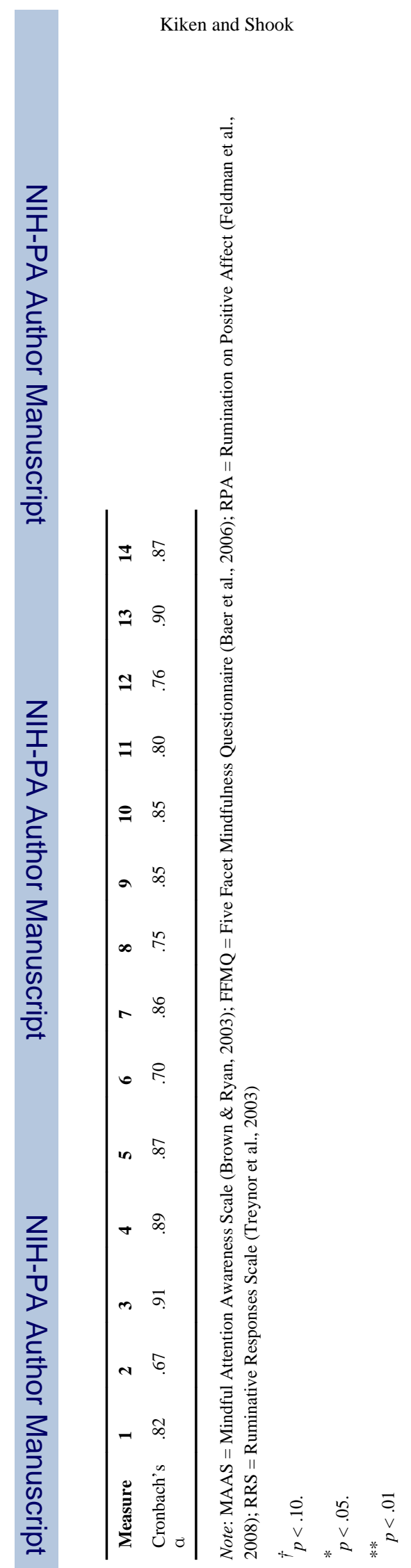

J Res Pers. Author manuscript; available in PMC 2015 December 01. 


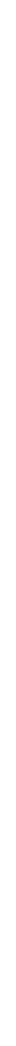

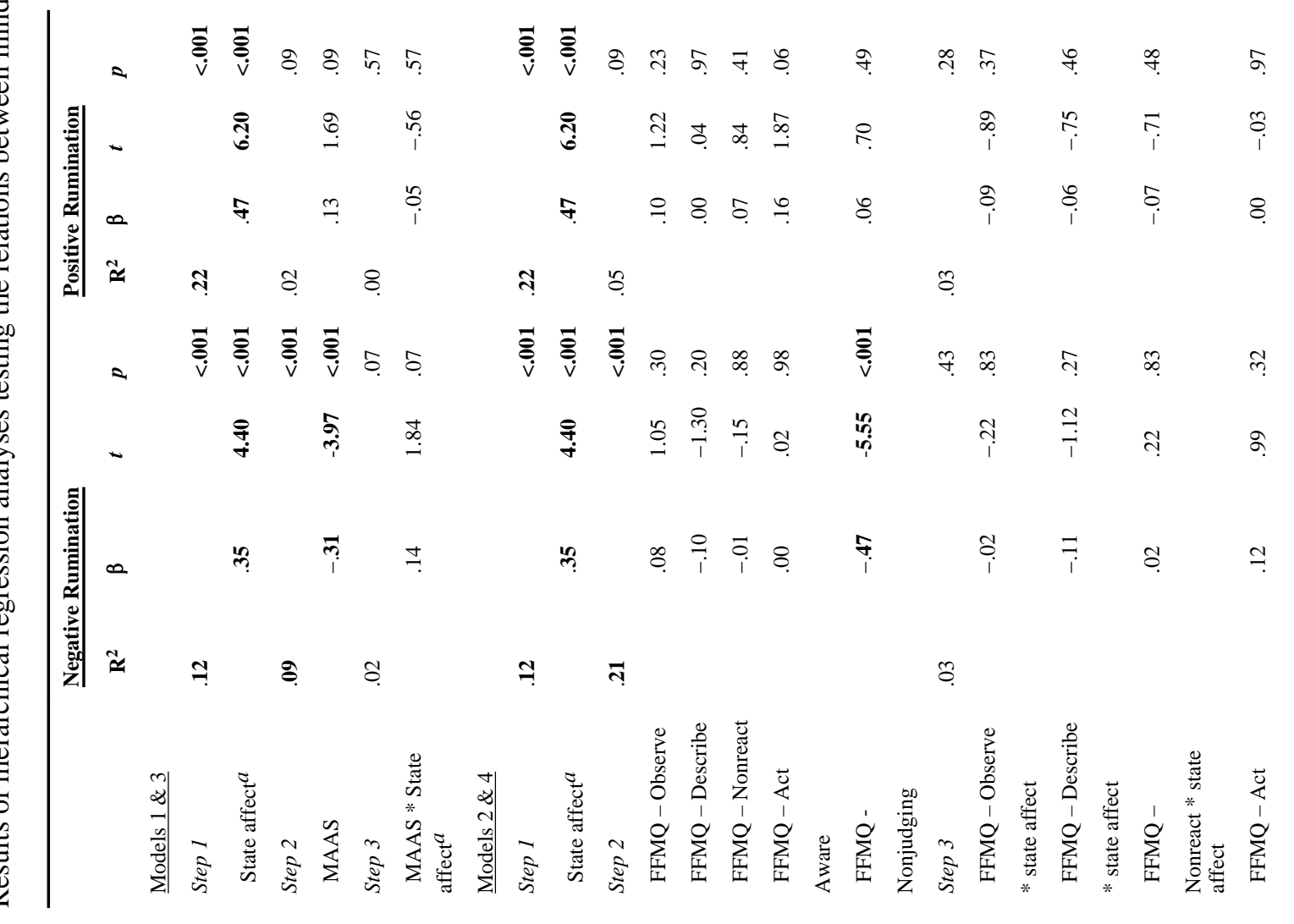

J Res Pers. Author manuscript; available in PMC 2015 December 01. 


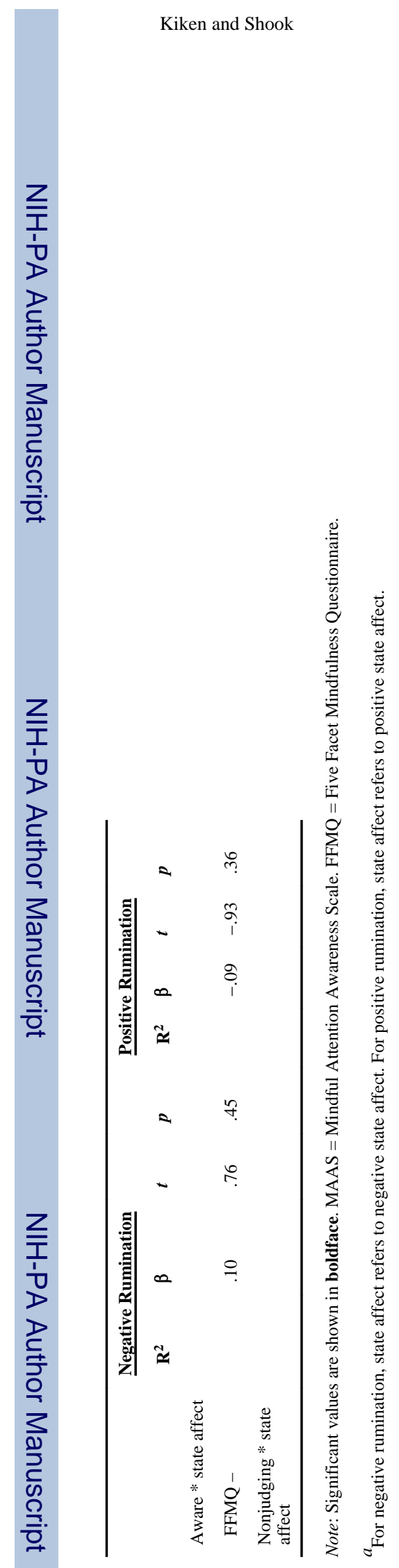

J Res Pers. Author manuscript; available in PMC 2015 December 01. 